\title{
A contribution to the knowledge of Euphorinae (Hymenoptera: Braconidae), with six new records from Iran
}

\author{
S. Farahani, ${ }^{1}$ A.A. Talebi, ${ }^{1}$ E. Rakhshani ${ }^{2}$ \\ ${ }^{1}$ Department of Entomology, Faculty of Agriculture, Tarbiat Modares University, Tehran; \\ ${ }^{2}$ Department of Plant Protection, College of Agriculture, University of Zabol, Iran
}

\begin{abstract}
A survey was conducted for identification of Euphorinae (Hymenoptera: Braconidae) in the northern provinces of Iran. The specimens were collected using Malaise traps during 2010-2011. In all, 9 species in four genera consisting of Allurus Förster, Dinocampus Förster, Peristenus Nees and Perilitus Nees were collected and identified. The genus Allurus is recorded for the first time from Iran. Six species are newly recorded for the Iranian fauna including Allurus muricatus (Haliday), Peristenus pallipes Curtis, Peristenus relictus (Ruthe), Perilitus (Townesilitus) bicolor (Wesmael), Perilitus foveolatus Reinhard and Perilitus rutilus (Nees). Morphological diagnostic characters and geographical distribution of the species are briefly discussed. A key is presented for identification of the genera and species in the studied area.
\end{abstract}

Correspondence: Ali Asghar Talebi, Department of Entomology, Faculty of Agriculture, Tarbiat Modares University, P.0. Box: 14115-336, Tehran, Iran. Tel.: +98.21.48292371 - Fax: +98.21.48292200.

E-mail: talebia@modares.ac.ir

Key words: Braconidae, Euphorinae, first records, Iran.

Acknowledgements: we would like to thank the Department of Entomology, Tarbiat Modares University for providing financial support. The authors are most grateful to the editor and anonymous reviewers for their valuable comments and suggestions on an earlier version of this paper. Our cordial thanks are also expressed to Dr. Cornelis van Achterberg for his cooperation in this work and identification of some species. We thank Mr. M. Khayrandish, A. Mohammadi and Mr. A. Nadimi for helping us in collecting the specimens.

Received for publication: 5 November 2012.

Revision received: 9 April 2013.

Accepted for publication: 30 May 2013.

(C) Copyright S. Farahani et al., 2013

Licensee PAGEPress, Italy

Journal of Entomological and Acarological Research 2013; 45:e9

doi:10.4081/jear.2013.e9

This article is distributed under the terms of the Creative Commons Attribution Noncommercial License (by-nc 3.0) which permits any noncommercial use, distribution, and reproduction in any medium, provided the original author(s) and source are credited.

\section{Introduction}

Euphorinae Förster, 1862 (Hymenoptera: Braconidae) is a rather large cosmopolitan subfamily of braconid wasps (van Achterberg \& Quicke, 2000). This subfamily comprises 55 genera worldwide of which 30 genera were found in the Palaearctic region (Tobias, 1995; Yu et al., 2012). van Achterberg (1994) recognized four tribes in this subfamily (Euphorini, Cosmophorini, Centistini, Meteorini), while Yu et al. (2012) listed 15 tribes (Centistini, Cosmophorini, Cryptoxilonini, Dinocampini, Euphorini, Helorimorphini, Mannokeraiini, Meteorini, Myiocephalini, Neoneurini, Oncometeorini, Perilitini, Proclithrophorini, Syntretini, and Tainitermini). A wide range of morphological variability within the subfamily Euphorinae, allow them to parasitize very different orders of insects including Coleoptera, Hemiptera, Psocoptera, Hymenoptera, Neuroptera and Orthoptera (Shaw \& Huddleston, 1991). Members of Euphorinae develop as solitary or gregarious endoparasitoids on larvae and adults of other insects (Shaw \& Huddleston, 1991; Belokobylskij, $2000 \mathrm{~b})$. This subfamily is readily recognizable by the following characters: having two submarginal cells in the forewing, first metasomal tergite distinctly petiolate (van Achterberg, 1993; Shaw \& Huddleston, 1991), spiracles of first metasomal tergite usually located medially or behind middle of the tergite, vein CUlb of forewing absent or nearly so (van Achterberg, 1993).

The taxonomy of the subfamily Euphorinae was studied by various authors (Loan, 1974; Shaw, 1985, 1996; Belokobylskij, 1992; van Achterberg, 1994; Chen \& van Achterberg, 1997; Haeselbarth, 1988, 1998, 1999; van Achterberg and Quicke, 2000; Shaw \& Marsh, 2000; Belokobylskij, 2000b; van Achterberg \& Haeselbarth, 2003; Boring, 2010; Stigenberg \& Ronquist, 2011). Comparatively, a considerable amount of data have been published about the biology of Euphorinae (Loan, 1983; Chen \& van Achterberg, 1997; Belokobylskij, 2000b; Maetô, 1988, 1990; Papp, 1994; Zijp \& Blommers, 2002; Waloff, 1967; Phillips \& Baird, 2001; Bilewicz-Pawinska, 1990).

The north of Iran is characterized by two different ecological zones that are separated by the Alborz Mountains: Hyrcanian (Caspian) (Figure 1) and Iran-o-Turanian zones. The Hyrcanian zone includes Alborz range forest steppe, Caspian mixed forest and Caspian lowland desert. The Iran-0-Turanian zone includes both mountains and plain areas dominated by a desert climate and a hot summer. These zones form diverse and vast regions of mountain, lush irrigated lowlands, wetland and desert with a great biodiversity, which is associated with the diverse flora, topographical irregularity and the xeric landscapes (Heshmati, 2007).

The subfamily Euphorinae in Iran is very poorly studied. Seven tribes and 20 species are listed (as genus-species) from Centistini (11), Dinocampini (1-1), Euphorini (3-5), Meteorini (2-6), Neoneurini (1-1), Perilitini (2-4) and Syntretini (1-2) (Fallahzadeh \& Saghaei, 2010; Ghahari et al., 2009a, 2009b, 2010; Ghahari \& Fischer, 2011; 
Lashkari-Bod et al., 2011; Farahani et al., 2012). The objective of this study was to determine the species of the subfamily Euphorinae, as a primary step to understand the situation of this large and diverse group of insects in northern provinces of Iran and to provide a reference collection at Tarbiat Modares University.

\section{Materials and methods}

The present study was carried out in four northern provinces including, Guilan, Mazandaran, Alborz and Qazvin provinces. The specimens were collected using 32 Malaise traps; 16 traps were set up in northern slopes and 16 traps in southern slopes of the Alborz Mountains in both ecosystems, during 2010 and 2011 (Figure 2). The specimens were extracted from the Malaise traps and sorted weekly. They were then treated with $70 \%$ ethanol and finally placed on a paper plate for drying. The dried specimens were then mounted on triangular papers and labeled. The external morphology of specimens were studied using an Olympus SZX9 stereomicroscope. Identifications were performed based on Loan (1974), Tobias (1995) and Haeselbarth (1999). Illustrations were taken using an Olympus SZX9 stereomicroscope and Olympus AX70 microscope equipped with a Sony CCD digital camera. Morphological terminology follows van Achterberg (1993). All the materials were deposited in the insect collection of the Department of Entomology, Tarbiat Modares University, Tehran.

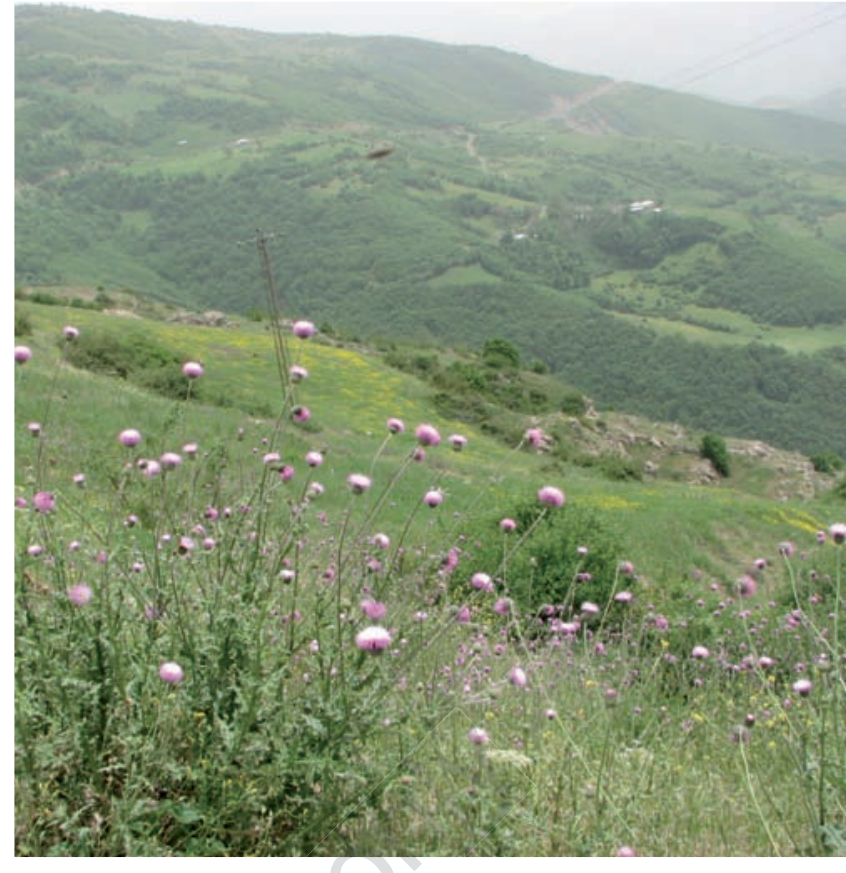

Figure 1. Habitats of Guilan province located in the northern slopes of the Alborz Mountain.

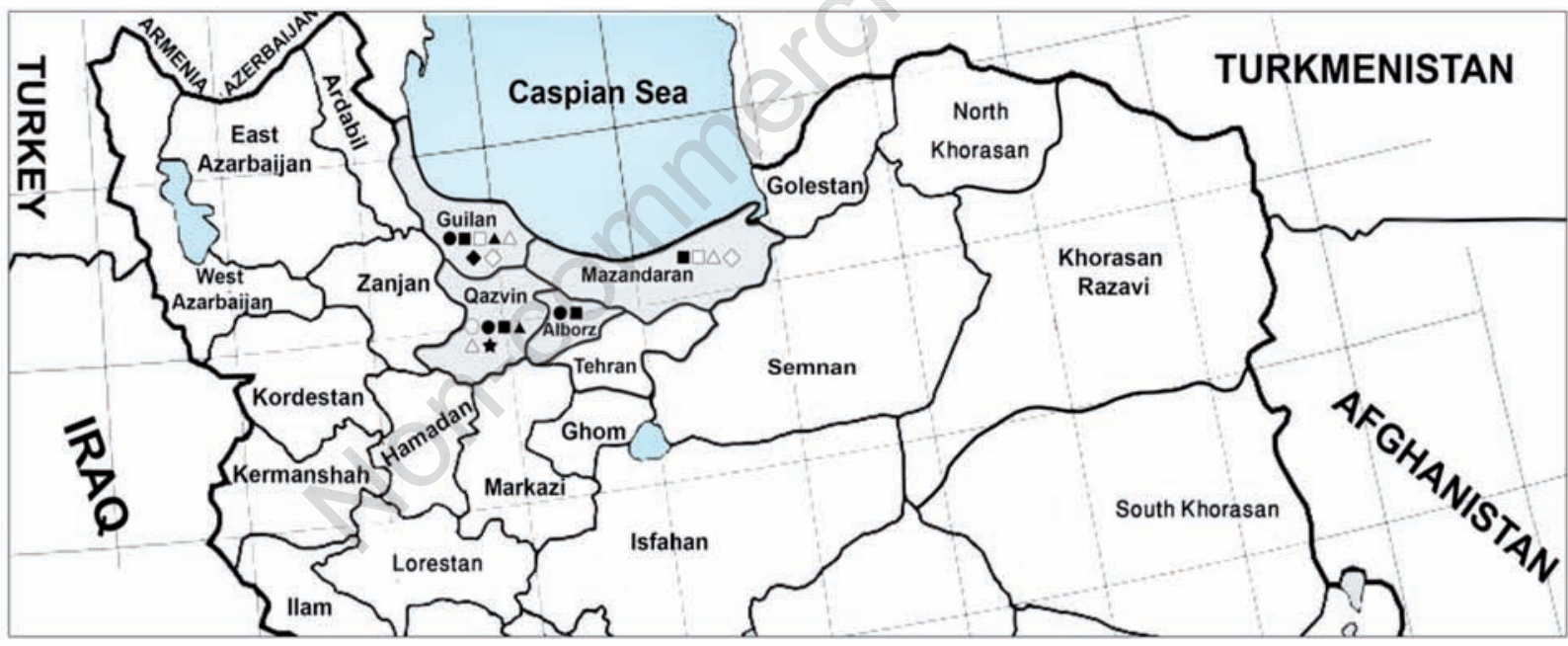

Allurus muricatus (Haliday, 1833): Qazvin province.

- Dinocampus coccinellae (Schrank, 1802): Alborz, Guilan and Qazvin provinces.

- Perilitus aethiops Nees, 1834: Alborz, Guilan, Mazandaran and Qazvin provinces.

$\square$ Perilitus bicolor (Wesmael 1835): Guilan and Mazandaran provinces.

- Perilitus foveolatus Reinhard 1862: Guilan and Qazvin provinces.

$\triangle$ Perilitus rutilus (Nees, 1811): Guilan, Mazandaran and Qazvin provinces.

- Perilitus stelleri (Loan, 1972): Guilan province.

$\diamond$ Peristenus pallipes Curtis, 1833: Guilan and Mazandaran provinces.

-Peristenus relictus (Ruthe, 1856): Qazvin province.

Figure 2. Iran-Alborz, Qazvin, Guilan and Mazandaran provinces, where the Euphorinae species were collected by Malaise trap. 


\section{Results}

Four genera including nine species of Euphorinae (Hym.: Braconidae) were collected and identified from northern Iran. They include three previously reported species, Perilitus aethiops Nees, Perilitus stelleri (Loan) and Dinocampus coccinellae (Schrank), and six newly recorded species for the Iranian fauna, Allurus muricatus Haliday, Peristenus pallipes Curtis, Peristenus relictus (Ruthe), Perilitus (Townesilitus) bicolor (Wesmael), Perilitus foveolatus Reinhard, Perilitus rutilus (Nees), which are marked with an asterisk in the text.

\section{*Allurus muricatus (Haliday, 1833) (Figures 3A-3E)}

SYNONYMS: Ancylus muricatus Haliday, 1833; Leiophron (Ancylus) muricatus Haliday, 1835; Leiophron muricatus Reinhard, 1862; Liophron muricatus Marshall, 1872; Centistes muricatus Rudow, 1918; Centistes (Allurus) muricatus Hellen, 1958; Allurus muricatus Forster, 1862; Leiophron armatus Wesmael, 1835.

MATERIAL EXAMINED: Qazvin province, Zereshk road $\left(36^{\circ} 21^{\prime} 39.72 ” \mathrm{~N}, 50^{\circ} 03^{\prime} 55.26\right.$ ”E, $1541 \mathrm{~m}$ a.s.l.), 24.5.2011, 1; 08.6.2011, 3 ㅇ; 21.6.2011, 1 今, leg. A. Nadimi.

DIAGNOSTIC CHARACTERS (FEMALE): Length of body 2.5-3.0 mm; antennae 30-31 segmented; length of fore wing 2.3-2.9 mm; pterostigma slightly shorter than vein 1-R1 (about $0.9 \times$ ); marginal cell long; vein $\mathrm{M}+\mathrm{CU1}$ unsclerotized; $1-\mathrm{SR}+\mathrm{M}$ and $2-\mathrm{SR}+\mathrm{M}$ of fore wing present (Figure 3B); first abdominal tergite sessile; third abdominal sternite with 2 denticles (Figure 3C); hind coax with a large denticle (Figure 3D); claws cleft (Figure 3E).

COLORATION: Antennae dark brown; head, thorax and first abdominal tergite black; second and third abdominal tergites reddish brown but reminder tergites black; legs reddish brown.

GENERAL DISTRIBUTION: Europe, Eastern and Western Palaearctic (Yu et al., 2012). New record from Iran.

Dinocampus coccinellae (Schrank, 1802) (Figures 4A, 4B)

SYNONYMS: Dinocampus americanus (Riley, 1888); Dinocampus sculptus (Cresson, 1872); Dinocampus terminatus (Nees, 1811).

MATERIAL EXAMINED: Alborz province, Shahriar (35\%40'08.01"N, 505'56.64"E, $1168 \mathrm{~m}$ a.s.l.), 10.9.2010, 1\%; Guilan province, Roodsar,

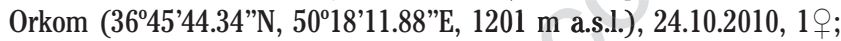
Qazvin province, Zereshk road $\left(36^{\circ} 21^{\prime} 39.72\right.$ 'N, 5003'55.26”E, $1541 \mathrm{~m}$ a.s.l.), 09.5.2011, 1 ; ; leg. A. Mohammadi.

DIAGNOSTIC CHARACTERS (FEMALE): Length of body $4.0 \mathrm{~mm}$; antennae 22-23 segmented; length of fore wing $3.2 \mathrm{~mm}$; pterostigma longer than vein 1-R1 (1.5×); marginal cell short; vein $M+C U 1$ sclerotized; 1-SR+M and 2-SR+M of fore wing present (Figure 4B); first abdominal tergite sessile; dorsope and laterope absent; ovipositor slender, about as long as first abdominal tergite; claws simple.

COLORATION: Antennae dark brown; head and fore legs reddish brown; thorax, first abdominal tergite, middle and hind legs black.

GENERAL DISTRIBUTION: Australasian, Europe, Nearectic, Neotropical, Oceanic, Oriental, Eastern and Western Palaearctic (Yu et al., 2012).

REMARKS: Dinocampus is small cosmopolitan genus with one species in the Palaearctic region, e.g. D. coccinellae (Chen \& van Achterberg, 1997).

Perilitus aethiops Nees, 1834 (Figures 5A, 6A, 7A, 8A)

SYNONYMS: Perilitus aethiopoides (Loan, 1975); Perilitus brevispina (Thomson, 1892); Perilitus spurius (Ruthe, 1856).

MATERIAL EXAMINED: Alborz province, Chalous Road, Shahrestanak (35'58'16.26”N, 51²1'25.80”E, $2225 \mathrm{~m}$ a.s.l.), 17.5.2010,

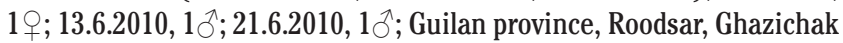

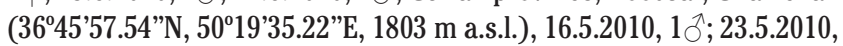

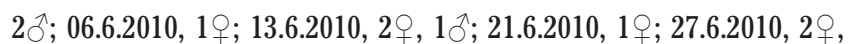

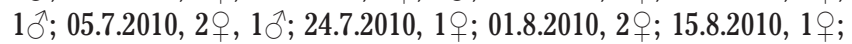

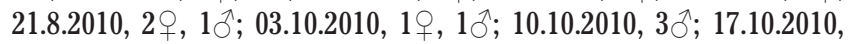
1ㅇ, 3今; 24.10.2010, 2犬; Guilan province, Roodsar, Orkom (36 ${ }^{\circ} 45^{\prime} 44.34 " \mathrm{~N}, 50^{\circ} 18^{\prime} 11.88^{\prime \prime} \mathrm{E}, 1201 \mathrm{~m}$ a.s.l.), 23.5.2010, 1 \% ; 05.7.2010, $1 \odot$; 24.10.2010, 2ᄋ; 31.10.2010, 1 ; Mazandaran province, Noor,

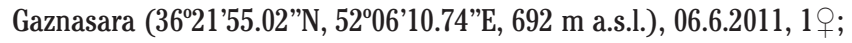
15.8.2011, 1 \% ; 04.9.2011, 1ð; Qazvin province, Zereshk road

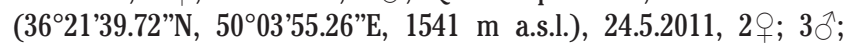

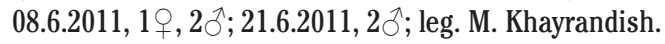

DIAGNOSTIC CHARACTERS (FEMALE): Length of body 1.9-3.0 mm; antennae 19-24 segmented, first flagellar segment $2.5 \times$ as long as wide (Figure 7A), second flagellar segment $2.0 \times$ as long as wide; face $1.3 \times$ wider than height (Figure 6A); length of fore wing 2.0-2.5 mm; pterostigma longer than vein 1-R1 $(2.0 \times)$; marginal cell short; vein $\mathrm{M}+\mathrm{CU1}$ sclerotized; $1-\mathrm{SR}+\mathrm{M}$ and $2-\mathrm{SR}+\mathrm{M}$ of fore wing absent (Figure $8 \mathrm{~A}$ ); first abdominal tergite petiolate, its length about $1.8 \times$ as long as apical width; ovipositor slender and equal or longer than first abdominal tergite; claws simple.

COLORATION: Antennae dark brown; head reddish brown, thorax and first abdominal tergite dark brown, occasionally reddish brown; legs reddish brown.

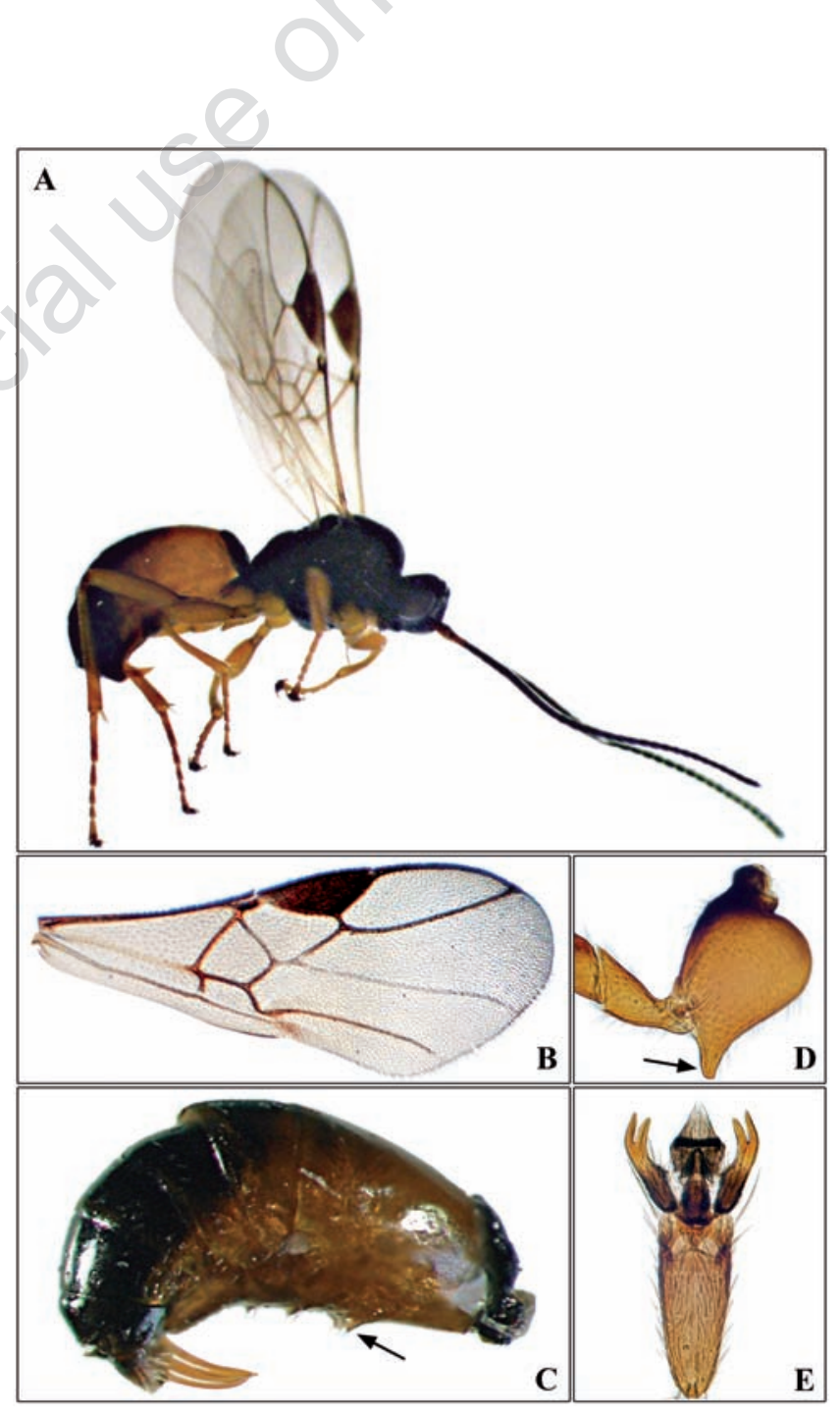

Figure 3. Allurus muricatus (Haliday, 1833), female: (A) lateral habitus; (B) fore wing; (C) abdomen (arrow); (D) hind coxa (arrow); (E) claw. 
GENERAL DISTRIBUTION: Ethiopian, Europe, Nearctic (introduced), Oriental, Eastern and Western Palaearctic (Yu et al., 2012).

REMARKS: This species seems to be nearest to P. stelleri, a form that can be distinguished by the apical width of the first abdominal tergite in the female (0.27-0.32) and width of the pterostigma (0.15-0.18), while the width of the first abdominal tergite $(0.30-0.43)$ and the width of the pterostigma (0.18-0.24) of the female in P. stelleri are relatively longer (Tobias, 1995).

*Perilitus (Townesilitus) bicolor (Wesmael 1835) (Figures 5B, 6B, 7B, 8B)

SYNONYMS: Perilitus (Townesilitus) breviradialis (Tobias, 1976).

MATERIAL EXAMINED: Guilan province, Roodsar, Ghazichak

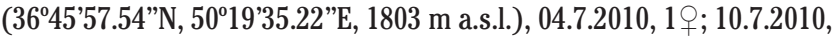
1 ; ; Guilan province, Roodsar, Orkom $\left(36^{\circ} 45^{\prime} 44.34^{\prime \prime} \mathrm{N}, 50^{\circ} 18^{\prime} 11.88^{\prime} \mathrm{E}\right.$, $1201 \mathrm{~m}$ a.s.l.), 16.5.2010, Mazandaran province, Noor, Gaznasara

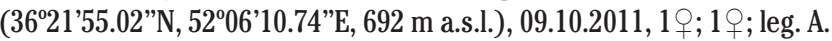
Nadimi.

DIAGNOSTIC CHARACTERS (FEMALE): Length of body 1.8-2.2 mm; antennae 18-21 segmented, flagellum long and slender, first flagellar segment 4.0-5.0× as long as wide (Figure 7B), second flagellar segment $4.0 \times$ as long as wide; face $1.1 \times$ wider than height (Figure $6 \mathrm{~B}$ ); length of fore wing 1.5-2.0 mm; pterostigma longer than vein 1-R1 $(2.0 \times)$; marginal cell short; vein $\mathrm{M}+\mathrm{CU} 1$ sclerotized; $1-\mathrm{SR}+\mathrm{M}$ and $2-\mathrm{SR}+\mathrm{M}$ of fore wing absent (Figure 8B); first abdominal tergite petiolate, its length about 3.0x as long as apical width; ovipositor slender and longer than first abdominal tergite; claws simple.

COLORATION: Antennae dark brown (basal of flagellum yellowish); head, thorax and first abdominal tergite reddish brown, occasionally dorsal part of thorax black; legs reddish brown.

GENERAL DISTRIBUTION: Europe, Eastern and Western Palaearctic (Yu et al., 2012). New record from Iran.

REMARKS: This species is easily recognized by the length of the first and second flagellar segment compared to their width, and antennae 18-21-segmented in the female (Tobias, 1995).

\section{*Perilitus foveolatus Reinhard 1862 (Figures 5C, 6C, 7C, 8C)}

SYNONYMS: Perilitus sicheli Giard, 1895.

MATERIAL EXAMINED: Guilan province, Roodsar, Orkom $\left(36^{\circ} 45^{\prime} 44.34\right.$ "N, $50^{\circ} 18^{\prime} 11.88^{\prime \prime} \mathrm{E}, 1201 \mathrm{~m}$ a.s.l.), 03.10.2010, 1 우; 17.10.2010, 19; Guilan province, Roodsar, Ziaz (36 ${ }^{\circ} 52^{\prime 2} 27.18$ ”N, $50^{\circ} 13$ '24.78”'E, 490 m a.s.l.), 30.5.2010, 1영 Guilan province, Roodsar, Ghazichak (36 45 '57.54"N, 50¹9'35.22”'E, $1803 \mathrm{~m}$ a.s.l.), 17.10.2010, 1 ; ; Qazvin province, Zereshk road $\left(36^{\circ} 21\right.$ '39.72”N, 50 03 '55.26”'E, $1541 \mathrm{~m}$ a.s.l.), 24.5.2011, 12 , 1 1 ; 08.6.2011, 1 \%; leg. M. Khayrandish.

DIAGNOSTIC CHARACTERS (FEMALE): Length of body 2.5-3.0 mm; antennae 22-23 segmented, first flagellar segment $3.5 \times$ as long as wide (Figure 7C), longer than second flagellar segment $(1.2 \times)$; face $1.1 \times$ wider than height (Figure 6C); length of fore wing 2.2-2.5 mm; pterostigma longer than vein 1-R1 (2x); marginal cell short; vein M+CU1 sclerotized; 1-SR+M of fore wing present and 2-SR+M absent (Figure 8C); apical area between notaulices distinctly punctuate; first abdominal tergite petiolate, its length about 1.9× as long as apical width; ovipositor slender and longer than first abdominal tergite; claws simple.

COLORATION: Antennae very dark brown; head lighter than thorax and reddish brown; thorax and first abdominal tergite black; legs reddish brown.

GENERAL DISTRIBUTION: Europe, Eastern and Western Palaearctic (Yu et al., 2012).

REMARKS: This species seems to be nearest to $P$. cornelii Haeselearth from which it can be recognized by length of ovipositor sheath, which is shorter than the hind tibia (Haeselbarth, 1999).

* Perilitus rutilus (Nees, 1811) (Figures 5D, 6D, 7D, 8D)

SYNONYMS: Perilitus luteus Herrich-Schaffer, 1838; Perilitus pyri
(Viereck, 1917); Perilitus ruralis Herrich-Schaffer, 1838; Perilitus strenuus Marshall, 1887; Perilitus tuberculus Zaykov, 1981.

MATERIAL EXAMINED: Guilan province, Roodsar, Orkom (36 ${ }^{\circ} 45^{\prime} 44.34$ "N, $50^{\circ} 18^{\prime} 11.88^{\prime \prime E}, 1201 \mathrm{~m}$ a.s.l.), 06.6.2010, 2 '; 13.6.2010, 3今; 21.6.2010, 19; 05.7.2010, 19; Guilan province, Roodsar, Ziaz

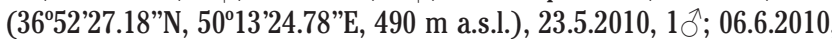
$1 \bigcirc$; Mazandaran province, Noor, Gaznasara (36 21 '55.02"N, $52^{\circ} 06^{\prime} 10.74$ 'E, $692 \mathrm{~m}$ a.s.l.), 27.6.2011, 1 ${ }^{\top}$; Qazvin province, Zereshk road $\left(36^{\circ} 21^{\prime} 39.72 ” \mathrm{~N}, 50^{\circ} 03^{\prime} 55.26^{\prime \prime} \mathrm{E}, 1541 \mathrm{~m}\right.$ a.s.l.), 24.5.2011, $2{ }^{\lambda}$; 08.6.2011, 4, 17 ; 21.6.2011, 4 ㅇ, 4 ; leg. M. Khayrandish.

DIAGNOSTIC CHARACTERS (FEMALE): Length of body $2.5-3.0 \mathrm{~mm}$; antennae 25-26 segmented, first flagellar segment $4.0 \times$ as long as wide (Figure 7D), first flagellar segment as long as or slightly shorter than second segment; face $1.4 \times$ wider than height (Figure 6D); length of fore wing 2.2-2.5 $\mathrm{mm}$; pterostigma longer than vein 1-R1 (1.3 $\times)$; marginal cell short and pointed apically; vein $\mathrm{M}+\mathrm{CU} 1$ sclerotized; $1-\mathrm{SR}+\mathrm{M}$ of fore wing present and 2-SR+M absent (Figure 8D); first abdominal tergite petiolate, its length about $2.5 \times$ as long as apical width; ovipositor slender and longer than first abdominal tergite; posterior area of hind coxa distinctly transverse striate; claws simple.

COLORATION: Antennae dark brown, occasionally basal segments of antennae lighter; body yellowish brown; propodeum black; first abdominal tergite at the base pale; legs yellowish brown.

GENERAL DISTRIBUTION: Europe, Nearctic (introduced), Eastern and Western Palaearctic (Yu et al., 2012).

REMARKS: This species is taxonomically similar to $P$. longiradialis, from which it can be separated by the basal felagellar segments, which are thin and yellowish; antennae 23-27-segmented (28-segmented in $P$. longiradialis) (Haeselbarth, 1999).

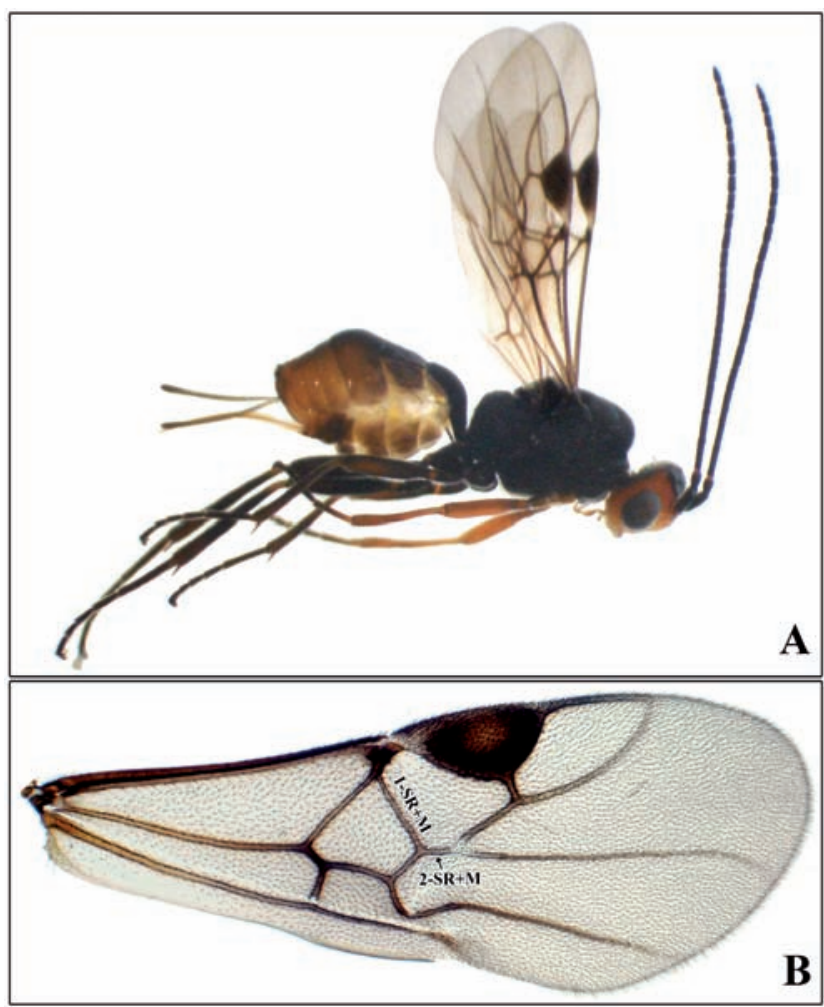

Figure 4. Dinocampus coccinellae (Schrank 1802), female: (A) lateral habitus; (B) fore wing. 
Perilitus stelleri (Loan, 1972) (Figures 5E, 6E, 7E, 8E)

MATERIAL EXAMINED: Guilan province, Roodsar, Orkom

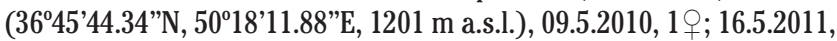
$1{ }^{\top}$; leg. A. Nadimi.

DIAGNOSTIC CHARACTERS (FEMALE): Length of body $3.4 \mathrm{~mm}$; antennae 25 segmented, length of first flagellar segment $3.0 \times$ as long as wide (Figure 7E), first segment about $1.3 \times$ as long as second segment; face $1.4 \times$ wider than height (Figure $6 \mathrm{E}$ ); length of fore wing 3.1 $\mathrm{mm}$; pterostigma longer than vein 1-R1 (1.6x); marginal cell short and pointed; vein $\mathrm{M}+\mathrm{CU1}$ sclerotized; $1-\mathrm{SR}+\mathrm{M}$ and $2-\mathrm{SR}+\mathrm{M}$ of fore wing absent (Figure 8E); first abdominal tergite petiolate; ovipositor slender and longer than first abdominal tergite; claws simple.

COLORATION: Body very dark brown; antennae dark brown, legs and ovipositor reddish brown.

GENERAL DISTRIBUTION: Europe, Western Palaearctic and introduced into USA (Yu et al., 2012).

\section{*Peristenus pallipes Curtis, 1833 (Figures 9A, 9B)}

SYNONYMS: Peristenus barbiger (Wesmael, 1835); Peristenus pallipes (Herrich-Schaffer, 1838); Peristenu punctata (Provancher, 1883); Peristenus tuberculifer (Marshall, 1887).

MATERIAL EXAMINED: Guilan province, Roodsar, Orkom

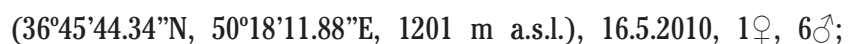
06.6.2010, 19; Guilan province, Roodsar, Ghazichak $\left(36^{\circ} 45^{\prime} 52.62 ” \mathrm{~N}\right.$, 5020'10.80”E, $1787 \mathrm{~m}$ a.s.l.), 16.5.2010, $1{ }^{\top}$; Guilan province, Roodsar, Ziaz (36 $52^{\prime} 27.18$ "N, 50¹3'24.78”E, $490 \mathrm{~m}$ a.s.l.), 30.5.2010, 1; Mazandaran province, Noor, Gaznasara $\left(36^{\circ} 16^{\prime} 58.08\right.$ ”N, $52^{\circ} 10^{\prime} 55.62$ ”E,

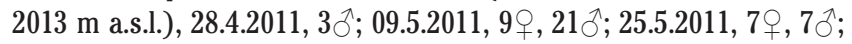
leg. M. Khayrandish.

DIAGNOSTIC CHARACTERS (FEMALE): Length of body 2.7-3.0 mm; antennae 23-segmented; occipital carina complete dorsally; frons densely punctuate; mesoscutum mostly punctated; fore wing vein 1-R1 $0.8 \times$ as long as width of pterostigma, pterostigma $2.2 \times$ as long as width; vein $\mathrm{M}+\mathrm{CU} 1$ of fore wing unsclerotized, basal cell of fore wing densely setose, distinctly similar to first subdiscal cell (Figure 9B); first abdominal tergite $1.7 \times$ as long as wide at apex; ovipositor shorter than first abdominal tergite and hardly visible.

COLORATION: Head and thorax uniformely black; antennae reddish brown; legs yellowish.

GENERAL DISTRIBUTION: Europe, Nearctic (Introduced), Oriental, Eastern and Western Palaearctic (Yu et al., 2012). New record from Iran.

REMARKS: This species is taxonomically nearest to P. nitidus, from which it can be recognized by its punctuated frons and roughened mesepisternum (Loan, 1974).
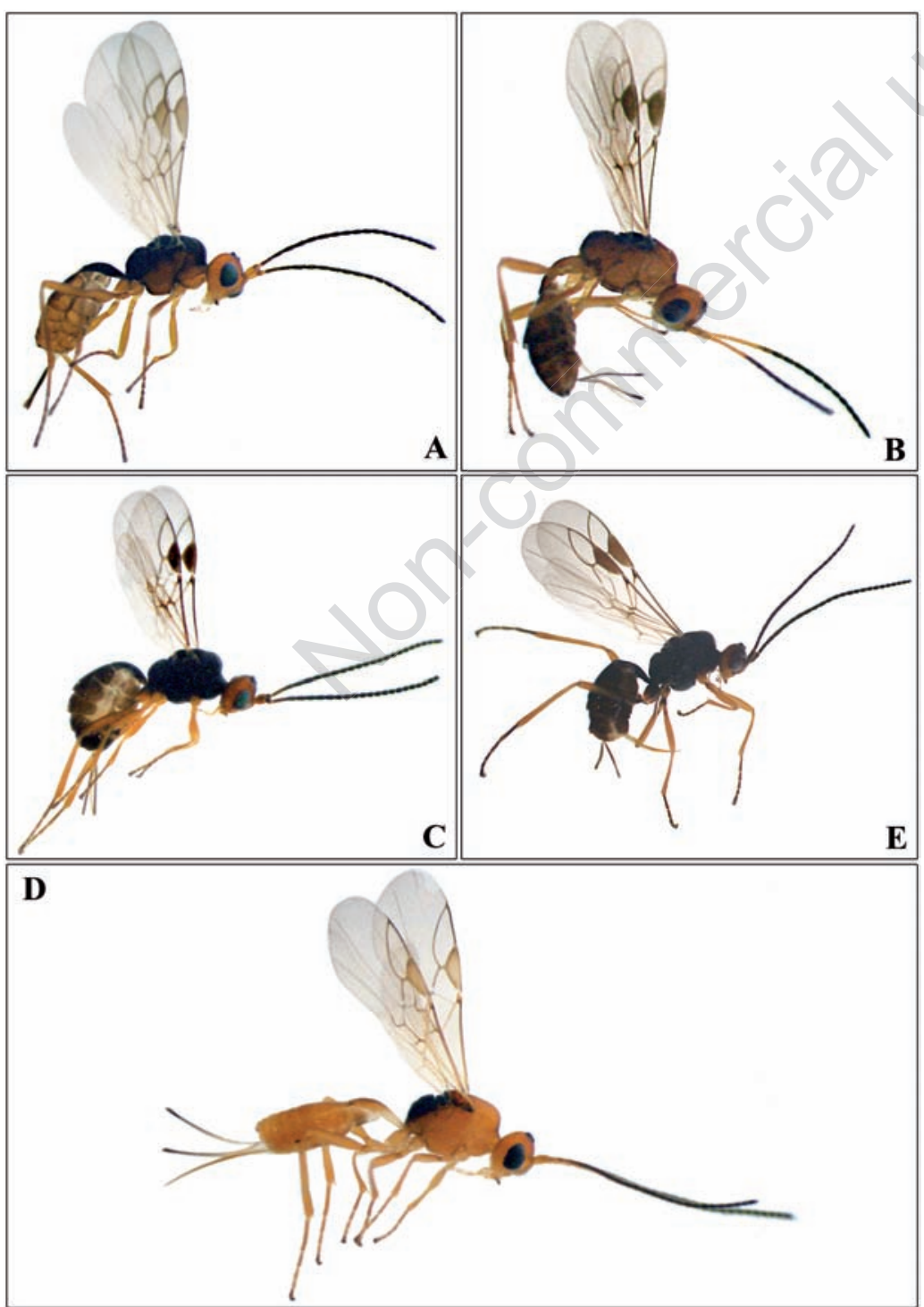

Figure 5. Lateral habitus of adult Perilitus species, females: (A) P. aethiops; (B) P. bicolor; (C) P. foveolatus; (D) P. rutilus; (E) P. stelleri. 
*Peristenus relictus (Ruthe, 1856) (Figures 9C, 9D)

SYNONYMS: Perilitus stygicus Loan, 1973.

MATERIAL EXAMINED: Qazvin province, Zereshk Road $\left(36^{\circ} 25^{\prime 23.88 ” N}\right.$, 5006’37.68”E, 1926 m a.s.l.), 06.7.2011, 2 ; ; leg. A. Mohammadi.

DIAGNOSTIC CHARACTERS (FEMALE): Length of body $2.7 \mathrm{~mm}$; antennae 19 segmented; occipital carina complete, thin and weak dorsally; frons punctate; mesoscutum smooth, notaulices of mesonotum sharply impressed, finely foveolatus; fore wing vein 1-R1 $0.6 \times$ as long as width of pterostigma, pterostigma $2.0 \times$ its width; vein $\mathrm{M}+\mathrm{CU} 1$ of

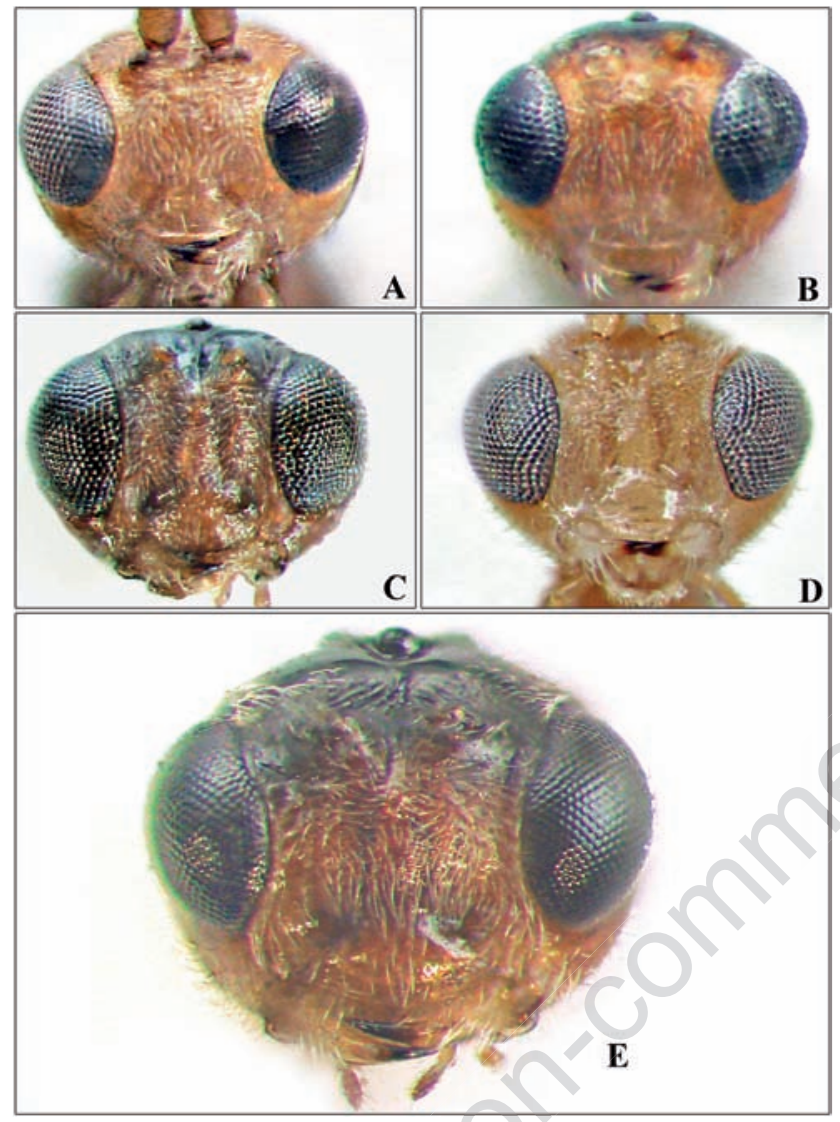

Figure 6. Frontal view of head in Perilitus species, females: (A) $P$ aethiops, (B) P. bicolor, (C) P. foveolatus, (D) P. rutilus, (E) P. stelleri.

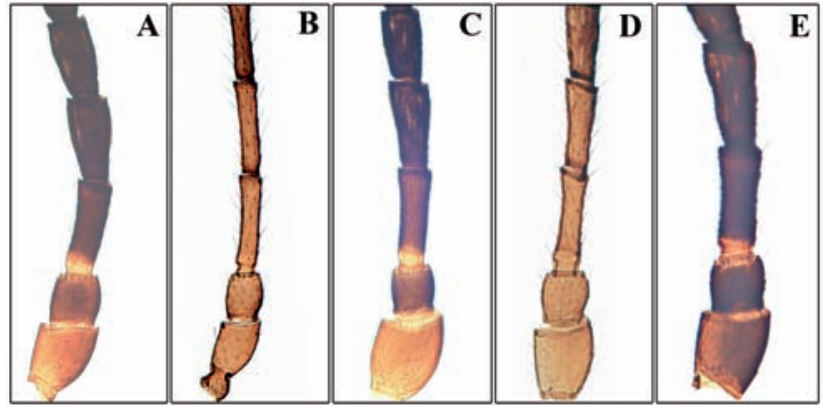

Figure 7. Basal antennal segments in Perilitus species: (A) P. aethiops; (B) P. bicolor; (C) P. foveolatus; (D) P. rutilus; (E) P. stelleri. fore wing unsclerotized, basal cell of fore wing sparsely setose or largely glabrous, distinctly less setose than first subdiscal cell (Figure 9D); first abdominal tergite $2.0 \times$ as long as wide at apex; ovipositor shorter than first abdominal tergite and hardly visible.

COLORATION: Body black; scape, pedicel and flagellomeres 1 and 2 usually reddish brown; fore leg reddish brown, mid and hind legs brownish black with base of tibia reddish brown.

GENERAL DISTRIBUTION: Europe, Western palaearctic and introduced in to Nearectic (Yu et al., 2012).

REMARKS: Peristenus relictus and $P$. stygicus were described as two

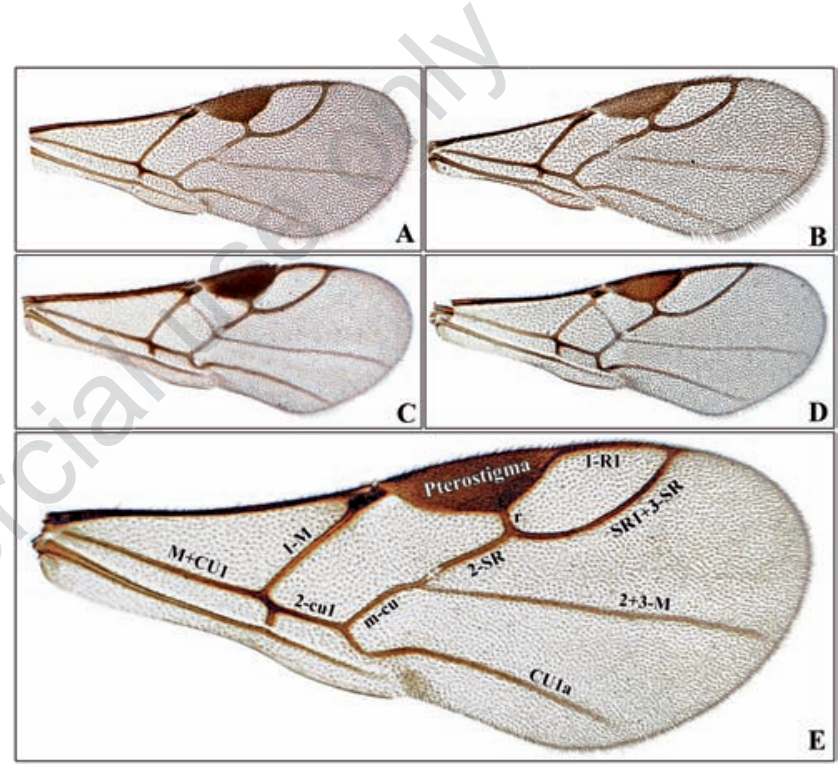

Figure 8. Fore wing in Perilitus species: (A) P. aethiops; (B) P. bicolor; (C) P. foveolatus; (D) P. rutilus; (E) P. stelleri.

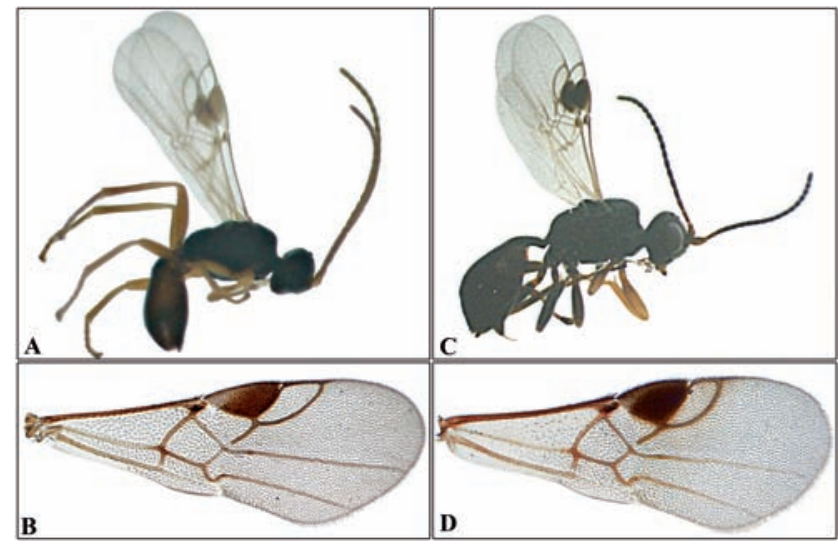

Figure 9. Lateral habitus of adult female and fore wing in Peristenus species: (A, B) P. pallipes; (C, D) P. relictus. 
valid species by Loan (1974), however, Varis \& van Achterberg (2001) have mentioned $P$. stygicus as a junior synonym of $P$. relictus. Perlitus relictus is taxonomically nearest to $P$. picipes, from which it can be separated by its punctuated frons and strong notaulices (Loan, 1974).

\section{Key to the genera and species}

Keys to the genera and species are the following: i)

- Vein 1-R1 of fore wing longer than pterostigma (Figure 3B); claws cleft (Figure 3E); third abdominal sternite with 2 denticles (Figure 3C); denticles on hind coxae large (Figure 3D): Allurus muricatus.

- Vein 1-R1 of fore wing shorter than pterostigma (Figure 4B, 8A-8E 9B, 9D); claws simple; third abdominal sternite and hind coxae without denticles: 2 .

ii)

- Vein M+CU1 of fore wing distinctly unsclerotized (Figure 9B, 9D); ovipositor shorter than first abdominal tergite and hardly visible (genus Peristenus Nees): 3.

- Vein M+CU1 of fore wing distinctly sclerotized (Figure 4B; 8A-8E); ovipositor longer than first abdominal tergite: 4 .

iii)

- Vein 1-R1 of fore wing $0.8 \times$ as long as width of pterostigma (Figure $9 \mathrm{~B})$; antennae of female 23 segmented; antennae and legs yellowish brown: Peristenus pallipes.

- Vein 1-R1 of fore wing $0.5 \times$ as long as width of pterostigma (Figure 9D); antennae of female 19 segmented; antennae, mid and hind legs brownish black: Peristenus relictus.

iv)

- Scutellum largely rugose; occipital carina complete, ventrally separated from hypostomal carina: Dinocampus coccinellae.

- Scutellum largely smooth; occipital carina complete, ventrally joined hypostomal carina (genus Perilitus Nees): 5

v)

- Vein 1-SR+M of fore wing absent (Figure 8A, 8B, 8E): 6 .

- Vein 1-SR+M of fore wing present (Figure 8C, 8D): 8.

vi)

- Basal segments of flagellum slender and 4.0-5.0x as long as width (Figure 7B), lighter than rest of flagellum (Figure 5B); antennae 1821 segmented: Perilitus bicolor.

- Basal segments of flagellum normal and almost $3.0 \times$ as long as width (Figure 7A, 7C, 7D, 7E); antennae 19-25 segmented: 7.

vii)

- Antenna of female 25 segmented (31 segmented of $\lesssim$ ); length of pterostigma 1.6× as long as 1-R1 (Figure 8E); length of first abdominal tergite $2.5 \times$ as long as apical width: Perilitus stelleri.

- Antenna of female 19-24 segmented (27-28 segmented in $\bigcirc$ ); length of pterostigma $2.5 \times$ as long as 1-R1 (Figure $8 \mathrm{~A}$ ); length of first viii) abdominal tergite $1.8 \times$ as long as apical width: Perilitus aethiops.

- Color of body ligth; first flagellar segment as long as second flagellar segment (Figure 7D); antennal of female 25-26 segmented; marginal cell pointed and apex of marginal cell closer to wing apex than stigma or their middle (Figure 8D): Perilitus rutilus.

- Color of body derk; first flagellar segment $1.2 \times$ as long as second flagellar segment (Figure 7C); antennal of female 22-23 segmented; marginal cell rounded and apex of marginal cell much closer to stigma than wing apex (Figure 8C): Perilitus foveolatus.

\section{Discussion and conclusions}

Nine species of Euphorinae were found in this study from northern provinces of Iran; of these, six species were newly recorded, which increased the number of known Euphorinae in Iran from 20 to 26. The first records of Perilitus from Iran were made by Hedwig (1957). Other researchers who studied the Perilitus fauna of Iran are Bartlett $e t$ al. (1978), Arbab and McNeill (2001) and Ghahari et al. (2010). Arbab and McNeill (2001) reported P. aethiops as a parasitoid that attacks adult alfalfa weevil (Hypera positica) from Qazvin and Hamadan provinces and Ghahari et al. (2010) has recorded $P$. stelleri from Isfahan province (cornfield). Mirab-balou et al. (2008) has reported one undetermined species of the genus Peristenus for the first time from Iran (Hamadan province). Lashkari-Bod et al. (2011) has recorded Peristenus picipes (Curtis) from Fars province. Fallahzadeh and Saghaei (2010) erroneously reported $P$. rubricollis (Thomson) with reference to Khanjani (2004).

The genus Allurus of the tribe Centistini is recorded here for the first time from Iran. Among the neighboring countries, Allurus muricatus has already been recorded from Kazakhstan (Tobias, 1995), while another species, A. lituralis (Haliday, 1835) has been recorded from Turkey (Yilmaz et al., 2010). The specimens were collected using Malaise traps in this study, therefore the biology of the recorded species are unknown. However, according to the previous studies, Allurus muricatus is considered as an adult parasitoid of Sitona sp. (Coleoptera: Curculionidae) and Stigmella sp. (Lepidoptera: Nepticulidae) species (Aeschlimann, 1980; Yu et al., 2012), but these records have been placed in doubtful hosts until they are confirmed by further investigations. This species is known to oviposit in adults and emerge from adult stages of the hosts (Aeschlimann, 1980). It was found only in Qazvin province from late May to late June 2011.

Dinocampus coccinellae is a solitary koinobiont endoparasitoid on Coccinellidae but it has also been recorded as attacking Chrysomelidae and Curculionidae (Yu et al., 2012). It parasitizes larvae, pupae and adults but only emerges from adults. Bagheri (1998) has reported Dinocampus coccinellae on Coccinella septempunctata L. (Coleoptera: Coccinellidae) from Isfahan. We also received some specimens of $D$. coccinellae from Yazd province as an endoparasitoid of C. septempunctata in April 2010.

Belokobylskij (2000a; 2000b) introduced Townesilitus as a subgenus of Perilitus. Many species of the genus Perilitus are recorded as parasitoids of Curculionoidea and Chrysomeloidea. Perilitus aethiops and $P$. stelleri are most commonly recorded as parasitoids of Curculionoidea (Yu et al., 2012). But Perilitus bicolor and $P$. foveolatus are commonly recorded as parasitoids of Chrysomeloidea. Perilitus rutilus is a solitary endoparasitoid and emerges from the adult stage of various genera of Coleoptera, such as Hylobius, Hypera, Pityokteines, Sitona and Tytthaspis. Many species of the genus Perilitus, as well as some other genera like Syntretus Forster, are gregarious parasitoids (Shaw and Huddleston, 1991). Perilitus foveolatus is a gregarious endoparasitoid on Timarcha Latreille (Coleoptera: Chrysomelidae) (Haeselbarth, 1999).

The genus Peristenus is most commonly recorded as a parasitoid of Miridae nymphs, but also rarely on Chrysomelidae, Cicadellidae, Melandryidae and Nitidulidae (Loan, 1980; Yu et al., 2012).

Some species of the genus Peristenus have been used as a biological control agent against some species of the genus Lygus and alfalfa plant bugs, Adelphocoris lineolatus (Hemiptera: Pentatomidae) (Clancy, 1968; Coulson, 1994), whereas most host records of the genus Perilitus involve coleopteran families (Chrysomelidae, Curculionidae, Coccinellidae and Tenebrionidae). Some species of the genus Peristenus have been used as biological control agents of Sitona and Hypera (Coleoptera: Curculionidae) and Phyllotreta (Coleoptera: Chrysomelidae) (Yu et al., 2012). Moreover, increasing cases of their regulatory impact on important plant pests have been reported, making the members of the genus Perilitus important biological control agents (Coulson, 1987; Haye, 2004).

\section{References}

AESCHLIMANN J.P., 1980 - The Sitona (Col.: Curculionidae) species occurring on Medicago and their natural enemies in the Mediterranean region. - Entomophaga. 25: 139-153. 
ARBAB A., MCNEILL M., 2001 - New report of Microctonus aethiopoides (Hym.: Braconidae) in Iran. - J. Entomol. Soc. Iran. 21: 111-112.

BAGHERI M.R., 1998 - The first report of Perilitus coccinellae (Hym., Braconidae) a parasitoid of Coccinella septempunctata in Isfahan. - Proc. $13^{\text {th }}$ Iranian Plant Protection Congress, Karaj, Iran, 200 pp.

BARTLETT B.R., CLAUSEN C.P., DEBACH P., GOEDEN R.D., LEGNER E.F., MCMURTRY J.A., et al., 1978 - Introduced parasites and predators of arthropod pests and weeds: A world review. Handbook No. 480. - Agricultural Research Service. US Dept. of Agriculture, Washington, D.C.: 545 pp.

BELOKOBYLSKIJ S.A., 1992 - Revision of the genus Centistes Haliday (Hymenoptera: Braconidae: Euphorinae) of USSR Far East and neighbouring territories. - Zool. Meded. Leiden. 66: 199-237.

BELOKOBYLSKIJ S.A., 2000a - Braconidae. In: LER P.A (ed.), Key to the insects of Russian Far East. Vol. IV. Neoropteroidea, Mecoptera, Hymenoptera. Part 4. Opredelitel nasekomykh Dalnego Vostoka Rossii. T. IV. Setchatokryloobraznye, skorpionnitsy, pereponchatokrylye. Ch.4. - Dalnauka, Vladivostok: 651 pp.

BELOKOBYLSKIJ S.A., 2000b - New species of the subfamily Euphorinae (Hymenoptera, Braconidae) from east Palaearctic. Far East. Entomol. 87: 1-28.

BILEWICZ-PAWINSKA T., 1990 - Response of parasitoids of the genus Peristenus Forster (Hymenoptera, Braconidae) to temperature changes during the diapause. - Entomol. Fennica. 1: 189-790.

BORING C.A., 2010 - Morphology and systematics of braconid wasps. PhD. Diss. - University of Kentucky, Lexington, KT: 123 pp.

CHEN X., VAN ACHTERBERGH C., 1997 - Revision of the subfamily Euphorinae (excluding the tribe Meteorini Cresson) (Hymenoptera: Braconidae) from China. - Zool. Verhandel. Leiden. 313:1-217.

CLANCY D.W., 1968 - Distribution and parasitization of some Lygus spp. in western United States and central Mexico. - J. Econ. Entomol. 61: $443-445$.

COULSON J.R., 1987 - Udies on the biological control of plant bugs (Heteroptera: Miridae): an introduction and history, 1961-1983. In: HEDLUND R. and GRAHAM H.M (eds.), Economic importance and biological control of Lygus and Adelphocoris in North America; ARS64. - USDA-ARS Natl. Techn. Informat. Serv., Springfield, VA, USA: $1-12$.

COULSON J.R., 1994 - Releases of beneficial organisms in the United States and Rerritories-1983; ARS-131. - Agricultural Research Service. US Dept. of Agriculture, Washington, D.C.: 113 pp.

FALLAHZADEH M., SAGHAEI N., 2010 - Checklist of Braconidae (Insecta: Hymenoptera) from Iran. - Mun. Ent. Zool. 5: 170-186.

FARAHANI S., TALEBI A.A., VAN ACHTERBERG C., RAKHSHANI E., 2012 - Syntretus, a genus of Euphorinae (Hymenoptera: Braconidae) new for Iran, with first record of two species. - J. Crop Prot. 1: 173-179.

GHAHARI H., FISCHER M., 2011 - A contribution to the Braconidae (Hymenoptera: Ichneumonoidea) from north-western Iran. Calodema. 134: 1-6.

GHAHARI H., FISCHER M., ERDOGAN 0.C., BEYARSLAN A., HAVASKARY M., 2009a - A contribution to the knowledge of the Braconid-fauna (Hymenoptera, Ichneumonoidea, Braconidae) of Arasbaran, northwestern Iran. - Entomofauna. 19: 329-336.

GHAHARI H., FISCHER M., ERDO AN Ö.Ç., TABARI M., OSTOVAN H., BEYARSLAN A., 2009b - A contribution to Braconidae (Hymenoptera) from rice fields and surrounding grasslands of Northern Iran. - Mun. Ent. Zool. 4: 432-435.

GHAHARI H., FISCHER M., HEDQVIST 0.C., ERDOGAN K., VAN ACHTERBERG K., BEYARSLAN A., 2010 - Some new records of Braconidae (Hymenoptera) for Iran. - Linzer biol. Beitr. 42: 1395-1404.

HAESELBARTH E., 1988 - Zur Braconidengattung Townesilitus Haeselbarth \& Loan, Entomofauna. 9: 429-460.

HAESELBARTH E., 1998 - Zur Braconiden-Gattung Perilitus NEES,
1818, 1. Beitrag: Die Perilitus falciger-Gruppe (Hymenoptera, Braconidae). - Entomofauna. 19: 197-208.

HAESELBARTH E., 1999 - Zur Braconiden-Gattung Perilitus Nees, 1818. Beitrag: Die Arten mit ausgebildetem ersten Cubitus-Abschnitt (Insecta, Hymenoptera, Braconidae). - Mitteil. Münch. Entomol. Ges. 89: 11-46.

HAYE T., 2004 - Studies on the ecology of European Peristenus spp. (Hymenoptera: Braconidae) and their potential for the biological control of Lygus spp. (Hemiptera: Miridae) in Canada. PhD. Diss. University of Kiel, Kiel: 182 pp.

HEDWIG K., 1957 - Ichneumoniden und Braconiden aus den Iran 1954 (Hymenoptera). -Jahresh. Ver. Vaterl. Naturkd. 112: 103-117.

HESHMATI G. A., 2007 - Vegetation characteristics of four ecological zones of Iran. - Int. J. Plant Prod. 1: 215-224.

KHANJANI M., 2004 - Field crop pests in Iran. - Bu-Ali Sina University press, Hamadan: 719 pp.

LASHKARI-BOD A., RAKHSHANI E., TALEBI A.A., LOZAN A., ZIKIC V., 2011 - A contribution to the knowledge of Braconidae (Hym., Ichneumonoidea) of Iran. - Biharean Biol. 5: 147-150.

LOAN C.C., 1974 - The European species of Leiophron Nees and Peristenus Foerster (Hymenoptera: Braconidae, Euphorinae). Trans. R. Ent. Soc. Lond. 126: 207-238.

LOAN C.C., 1980 - Plant bug hosts (Hymenoptera: Miridae) of some Euphorine parasites (Hymenoptera: Braconidae) near Belleville, Ontario, Canada. Naturaliste Canadien. 107: 87-93.

LOAN C.C., 1983 - Host and generic relations of the Euphorini (Hymenoptera: Braconidae). - Contrib. Am. Entomol. Inst. 20: 388-397.

MAETÔ K., 1988 - Systematic studies on the tribe Meteorini (Hymenoptera: Braconidae) from Japan. III. The hirsutipes group of the genus Meteorus Haliday. - Kontyu 56: 321-329.

MAETÔ K., 1990 - Phylogenetic relationship and host associations of the subfamily Meteorinae Cresson (Hymenoptera, Braconidae). Japan J. Entomol. 58: 383-396.

MIRAB-BALOU M., RASOULIAN G. R., KHANJANI M., SABAHI Q., 2008 Study on taxonomy of phytophagous bugs of the family Miridae and introducing insects natural enemies of the alfalfa tarnished plant bug in Hamedan alfalfa farms (West of Iran). - Pak. Entomol. 30: 55-60.

PAPP J., 1994 - The dispersion of braconid wasps in an oak forest of Hungary (Hymenoptera: Braconidae). - Folia Ent. Hung. 55: 305-320.

PHILLIPS C.B., BAIRD D.B., 2001 - Geographic variation in egg load of Microctonus hyperodae Loan (Hymenoptera: Braconidae) and its implications for biological control success. - Biocontrol Sci. Techn. 11: $371-380$

SHAW S.R., 1985 - A phylogenetic study of the subfamilies Meteorinae and Euphorinae (Hymenoptera: Braconidae). - Entomography. 3: 277-370.

SHAW S.R., 1996 - Plynops, a peculiar new genus and ten new species in the tribe Euphorini (Hymenoptera: Braconidae: Euphorinae). J. Hymenopt. Res. 5: 166-183.

SHAW M.R., HUDDLESTON T., 1991 - Classification and biology of Braconid Wasps (Hymenoptera: Braconidae). - British Museum of Natural History, London: 126.

SHAW S.R., MARSH P.M., 2000 - Revision of the enigmatic genus Marshiella Shaw in the New World with the description of three new species (Hymenoptera: Braconidae: Euphorinae). - J. Hymenopt. Res. 9: 277-287.

STIGENBERG J., RONQUIST F., 2011 - Revision of the West Palaearctic Meteorini (Hymenoptera, Braconidae), with a molecular characterization of hidden Fennoscandian species diversity. - Zootaxa. 3084: 1-95.

TOBIAS V.I., 1995 - Subfamily Euphorinae. In: MEDVEDEV G.S. (ed.), Keys to the insects of the European part of the USSR, vol. III, 
Hymenoptera Part IV. Amerind Publishing Co., New Delhi: 883 pp.

VAN ACHTERBERG C., 1993 - Illustrated key to the subfamilies of the Braconidae. (Hymenoptera: Ichneumonoidea). - Zool. Verhand. Leiden. 283: 1-189.

VAN ACHTERBERG C., 1994 - The Palaearctic species of the genus Chrysopophthorus Goidanich (Hymenoptera: Braconidae, Euphorinae). - Zool. Meded. Leiden. 68: 301-307.

VAN ACHTERBERG C., HAESELBARTH E., 2003 - Revision of the genus Syntretus Foerster (Hymenoptera: Braconidae: Euphorinae) from Europe. - Zool. Meded. Leiden. 77: 9-78.

VAN ACHTERBERG C., QUICKE D.L. J., 2000 - The Palaeotropical species of the tribe Cosmophorini Capek (Hymenoptera: Braconidae: Euphorinae) with descriptions of twenty-two new species. - Zool. Meded. Leiden. 74: 283-338.

VARIS A.L., VAN ACHTERBERG C., 2001 - Peristenus varisae spec. nov. (Hymenoptera: Braconidae) parasitizing the European tarnished plant bug, Lygus rugulipennis Poppius (Heteroptera: Miridae). Zool. Med. Leiden. 75: 371-380.

WALOFF N., 1967 - Biology of three species of Leiophron (Hymenoptera: Braconidae, Euphorinae) parasitic on Miridae on broom. - Trans. R. Entomol. Soc. Lond. 119: 187-213.

YILMAZ T., AYDOGDU M., BEYARSLAN A., 2010 - The distribution of Euphorinae wasps (Hymenoptera: Braconidae) in Turkey, with phytogeographical notes. - Turk. J. Zool. 34: 181-194.

YU D.S., VAN ACHTERBERG C., HORSTMANN K., 2012 - World Ichneumonoidea 2011. Taxonomy, biology, morphology and distribution (Braconidae). - Taxapad (Scientific Names for Information Management) Interactive Catalogue, Ottawa. Available from: http://www.taxapad.com

ZIJP J.P., BLOMMERS L.H.M., 2002 - Biology of Centistes delusorius, a parasitoid of adult apple blossom weevil. - Agric. For. Entomol. 4: 275-282. 